\title{
Constraints on the evolution of attractive traits: genetic (co)variance of zebra finch bill colour
}

\author{
DONALD K. PRICE* \& NANCY TYLER BURLEY† \\ Department of Ecology, Ethology, and Evolution, University of Illinois, Urbana, II 61801, U.S.A.
}

\begin{abstract}
We estimated the heritability and genetic correlation between male and female bill colour in a laboratory population of zebra finches (Taeniopygia guttata) in order to examine the potential genetic constraints on the evolution of a sexually dimorphic trait. The heritability estimates of bill colour from regressions of offspring on single parents ranged from $h^{2}=0.34$ to 0.73 and all but one of these estimates were significantly greater than zero. The restricted maximum likelihood heritability estimates for full- and half-siblings were significant for females $\left(h^{2}=0.48\right)$ but not significant for males $\left(h^{2}=0.45\right)$. The maximum likelihood estimates indicate that there is little dominance genetic variance for bill colour. The large genetic correlation between male and female bill colour $\left(r_{\mathrm{g}}=0.91\right)$ combined with opposing selection on male and female bill colour indicates that the evolution to sex-specific optima may proceed very slowly.
\end{abstract}

Keywords: attractive traits, bill colour, genetic covariance, heritability, sexual dimorphism, zebra finch.

\section{Introduction}

The evolution of sexual dimorphism has been explained as a consequence of natural selection and sexual selection, both independently and together (Selander, 1972; Lande, 1980a; Slatkin, 1984). The models usually require that there be differing selection on the trait when it is expressed in males and females (but see Cheverud et al., 1985) and that the genetic correlation of the trait in males and female is less than unity. Sexual dimorphism may arise in species in which either selection on the male trait is opposed by selection on the female trait or selection on the trait occurs in only one sex. In addition, the strength of the genetic correlation between the traits in the two sexes can dramatically alter both the evolutionary trajectories of the trait means expressed by males and females and the overall adaptiveness of sexually dimorphic traits (Lande, 1981, 1987). In birds, where the evolution of sexual dichromatism has received considerable attention (Darwin, 1871; Selander, 1972; Payne, 1984), the

Correspondence: D. K. Price.

*Present address: Department of Biology, University of Oregon, Eugene, OR 97403, U.S.A.

$\nmid$ Present address: Department of Ecology and Evolutionary Biology, University of California at Irvine, Irvine, CA 92717, U.S.A. feasibility of laboratory rearing allows tests of hypotheses regarding the genetic control of sexual dichromatism.

The sex-specific optima for sexually dichromatic traits in birds will be determined by the relative strengths of opposing sexual and natural selection (Lande, 1980a; Lande \& Arnold, 1985). The most likely sexual selection agents favouring bright coloration in male birds are the choice of mates by females and competition among males (Selander, 1972; Payne, 1984; Butcher \& Rohwer, 1989). Females choosing males that possess bright colours may receive direct benefits, such as offspring care, from brightly coloured males and this could lead to the increased expression of both female preferences and male colour traits (Pomiankowski, 1988; Kirkpatrick \& Ryan, 1991). Likewise, the genetic covariances between female preferences and male colour traits may also allow evolutionary elaboration of male and female traits even when females do not receive direct benefits from brightly coloured males (Fisher, 1958; Lande, 1981, 1987; Kirkpatrick, 1987). Selection for bright colour may also occur if the signalling of dominance status by colour is advantageous in male competition for access to females or to resources (Butcher \& Rohwer, 1989). The two types of natural selection that are most likely 
to oppose evolutionary increases in colour expression are predation and the physiological costs of colour expression.

The magnitude of the genetic variances and the magnitude and sign of the genetic covariances between sexually dimorphic traits are of critical importance in determining the consequences that selection in one sex will have on the direct or indirect evolutionary response to selection in the other sex. Additive genetic variance is required for an evolutionary response to selection and thus its existence is an assumption of all quantitative genetic models of sexual selection (Lande, 1980a, 1987). The level of genetic variance for attractive traits will be determined by a balance between constant selective pressures that tend to decrease genetic variance and fluctuating selective pressures and spontaneous mutations that tend to maintain genetic variance (Fisher, 1958; Charlesworth, 1988; Lynch, 1988). The genetic covariance between the sexes for the same trait is dependent on the existence of additive genetic variation for the trait in both sexes. Little is known about the strength of the genetic covariance between the sexes for attractive traits, but pleiotropic mutations may maintain genetic covariances for long time periods (Lande, 1980b).

The importance of genetic covariances between males and females for the evolution of attractive traits has received little empirical attention (Lande, 1987). Genetic covariances between the sexes have been reported for various morphological traits in mice (Eisen \& Legates, 1966; Eisen \& Hanrahan, 1972), friut flies (Harrison, 1953; Frankham, 1968a, 1968b; Crowley et al., 1986; Crowley \& Atchley, 1988) and humans (Rogers \& Mukherjee, 1992). The occurrence of relatively large genetic covariances between the sexes in these animals indicate that the selective pressures in one sex would have a large effect on the other sex and thus the evolution to sex-specific optima may proceed very slowly (Lande, 1980a; Lande \& Arnold, 1985). However, to date no published studies have estimated both the genetic covariances between the sexes and the nature of selection in males and females within a single species. Moreover, there is virtually no evidence regarding the strength of the genetic covariance between the sexes for avian colour traits.

In this paper, we examine the genetic variance of zebra finch bill colour in each sex and the genetic covariance between male and female bill colour. Females prefer males with the reddest bills and this preference extends beyond the typical male phenotypic range (Burley \& Coopersmith, 1987). Consistent with this female preference, males with the reddest bills had higher reproductive success in an experimental laboratory population (Price, 1991). In contrast, female bill colour is negatively correlated with reproductive success and this is primarily due to the decreased survival of redder-billed females (Price, 1991). The evidence for opposing phenotypic selection on male and female bill colour led us to hypothesize that the rate of evolution of bill colour in males and females is constrained by genetic factors. Therefore, we examined the genetic variance of bill colour and the genetic covariance between male and female bill colour in a laboratory population of zebra finches.

\section{Methods}

\section{Study organism}

The zebra finch is an ideal avian species for studying selection and genetic variance for a wide variety of traits. Zebra finches breed opportunistically year round. They become sexually mature at approximately 80 days after hatching (Immelmann, 1965) at which age they express adult plumage colours and bill colour (Burley \& Coopersmith, 1987). The generation time in the laboratory can be as short as 4 months, although in the wild typical generation times may be substantially longer. Zebra finches are semi-colonial breeders and defend only the immediate nest site (Immelmann, 1965; Goodwin, 1982). As a result they can be housed at relatively high densities in bisexual groups, permitting birds to interact and choose mates freely. The absence of a resource-based mating system suggests that sexual selection will be primarily directed toward the morphological or behavioural attributes of individual males, such as bill or plumage colour.

\section{Experimental design and data collection}

The general maintenance of the colony and the selection of individuals to begin this experiment is described in greater detail elsewhere (Price, 1991). The zebra finches used in this experiment were descendants of birds raised in large free-flight aviaries in similar laboratory conditions. We selected founders from the two extremes of the bill colour range to increase the statistical power for detecting both directional selection and genetic variance of bill colour (criteria for measuring bill colour are described below) (Hill \& Thompson, 1977). The selection of phenotypically extreme individuals does not appreciably bias estimates of genetic parameters unless there are loci that have large non-additive effects (Reeve, 1961). Fifteen males with the reddest and 15 males with the least red (most orange) bills were selected for the experiment from a base population of 94 males. Thirty females were selected in a similar manner from a base 
population of 72 females. For each sex, the base population consisted of a pool of adults produced in other experiments and maintained in unisexual groups until their inclusion in this experiment.

Each adult wore a numbered aluminium band. After the experiment had been in progress for 3 months each bird also received three leg bands of colours previously demonstrated not to influence mate choice (Burley, 1985). Parents were determined by observing colour banded adult birds involved in parental activities from an adjacent darkened room or occasionally by catching adults at the nest. Nestlings were given a numbered aluminium band for individual identification at about 10 days after hatching. Young were removed from the aviary and housed in unisexual groups under standard conditions. Breeding was stopped after 19 months by removing nest sites from the aviary.

The Munsell ${ }^{\circledR}$ colour system was used to measure bill colour and the three dimensions (hue, value and chroma) were converted to a single numerical colour dimension (Price, 1991; Burley et al., 1992). This single colour score produced large repeatabilities of zebra finch colour over both short and long periods (Burley et al., 1992). Bill colour was measured on the adults prior to the initiation of the experiment. Offspring bill colour was measured at 120 days after hatching (approximately 40 days after reaching sexual maturation).

\section{Data analysis}

Bill colour heritabilities were estimated from both regressions of offspring on parents and maximum likelihood analyses of full- and half-siblings. Offspring-parent genetic estimates were computed using the mean bill colour score for all offspring reared by a particular adult to control for ongoing selection. Since the phenotypic variance differed between males and females (Table 1), only genetic estimates based on single parents were computed (Falconer, 1981). Estimates of bill colour heritability and the associated standard error estimates are twice the slope and standard error, respectively, of the regression of offspring bill colour on adult bill colour. The ratio of the male and female bill colour standard deviations in the reproductive founders was used to adjust the daughter-father and son-mother heritability estimates (male:female standard deviation ratio $=0.77$ ) (Falconer, 1981). The heritability estimates were also adjusted for the weak negative assortative mating for bill colour using the formula $h^{2 *}=h^{2}(1+r)$, where $h^{2}$ is the corrected heritability estimate, $h^{2^{*}}$ is the uncorrected heritability estimate and $r$ is the phenotypic correlation between mates (Falconer, 1981). A separ- ate phenotypic correlation between mates was computed for each heritability estimate, because not all adult pairs were involved in each heritability estimate owing to the occurrence of single-sexed broods. The correlation between parents for each type of heritability estimate is as follows. Father-son: $r=-0.15$; father-daughter: $r=-0.23$; mother-son: $r=-0.18$; mother-daughter: $r=-0.05$ (none were statistically significant: $\boldsymbol{P}>0.1$ ).

The complex pedigree of the offspring produced in this experiment does not permit a standard leastsquares calculation of full- or half-sibling heritabilities. Approximately 40 per cent (41 of 103) of male offspring and 33 per cent ( 34 of 102) of female offspring, with an identified mother and father (see above), had at least one same-sexed half-sibling and one full-sibling. The other 60 per cent of male and 67 per cent of female offspring had only full-siblings. Therefore, we employed a maximum-likelihood (ML) program designed to calculate heritabilities from data with complex pedigrees (Shaw, 1987). In the maximum likelihood analyses the bill colour of parents was not included in the analysis of offspring bill colour because the bill colour phenotypic variances were substantially different between the parental and offspring generations due to selection of adults from the two extremes of the bill colour range (Table 1). This ML program calculates additive genetic, dominance genetic and environmental variance. The heritability estimate from the ML analysis is the additive genetic variance divided by the total phenotypic variance. The proportion of variance explained by dominance genetic variance is computed by dividing the dominance genetic variance by the total phenotypic variance. In the $M L$ analyses the dominance genetic variance was estimated to be negative for both male and female bill colour (see results). Therefore, restricted maximum likelihood (REML) analyses were employed by restricting the dominance genetic variance to zero and recalculating the other variances. The significance of the heritabilities (additive genetic variance) was obtained by computing twice the difference in long-likelihoods between a model where the additive genetic variance was constrained to be zero and a model where it was unconstrained (Shaw, 1987).

The genetic correlation coefficient between male and female bill colour was computed from the offspring-parent covariance estimates using the formula

$r_{\mathrm{g}}=\left(\operatorname{cov}_{\mathrm{mf}}+\operatorname{cov}_{\mathrm{fm}}\right) / 2 \sqrt{\left(\operatorname{cov}_{\mathrm{mm}}\right)\left(\operatorname{cov}_{\mathrm{ff}}\right)}$,

where $\operatorname{cov}_{\mathrm{ij}}$ is the additive genetic covariance estimates for bill colour in offspring of sex $i$ and parent of sex $j$.

The standard error of $r_{\mathrm{g}}$ was computed using the formula 
S.E. $\left(r_{\mathrm{g}}\right)=\left[\left(1-r_{\mathrm{g}}^{2}\right) / \sqrt{2}\right] \cdot\left[\sqrt{\left[\text { S.E. }\left(h_{\mathrm{mm}}^{2}\right)\right]\left[\text { S.E. }\left(h_{\mathrm{ff}}^{2}\right)\right] /\left(h_{\mathrm{mm}}^{2}\right)\left(h_{\mathrm{ff}}^{2}\right)}\right]$,

where $h_{\mathrm{mm}}^{2}$ and $h_{\mathrm{ff}}^{2}$ are the heritabilities of male and female bill colour and S.E. $\left(h_{\mathrm{mm}}^{2}\right)$ and S.E. $\left(h_{\mathrm{ff}}^{2}\right)$ are the standard errors of male and female bill colour heritability estimates (Falconer, 1981; Becker, 1985).

\section{Results}

\section{Phenotypic variance}

Male bill colour was significantly redder than female bill colour (Table 1). The selection of founder males and females with bill colours from the two ends of the phenotypic range changed the mean only slightly, but greatly increased the variances of male and female bill colour (Table 1). The founders that produced at least one offspring had means and variances of bill colour similar to those for the bill colours of all founders. The means and variances of the bill colour of 120-day-old offspring were similar to the adult bill colour in the base population. In both the parental and offspring generation, the variance in female bill colour exceeded the variance in male bill colour. Therefore, regressions of offspring bill colour on the bill colour of single

Table 1 Means and variances of adult and offspring bill colour. The 30 male and 30 females founders were chosen from the base population (see Methods). The reproductive founders are defined as those individuals that produced at least one fledgling. The bill colour of the founders' offspring was measured when they were adults ( 120 days post-

hatching). Differences between the mean bill colour of males and females are highly significant for all groups (ANOVA:

$*-P<0.01)$

\begin{tabular}{lcccc}
\hline & Mean & Variance & $N$ \\
\hline $\begin{array}{l}\text { Base population } \\
\text { Male }\end{array}$ & 24.95 & 4.18 & 94 \\
$\begin{array}{l}\text { Female } \\
\text { Founders } \\
\text { Male }\end{array}$ & 18.62 & 10.12 & 72 \\
$\begin{array}{l}\text { Female } \\
\text { Reproductive founders } \\
\text { Male }\end{array}$ & 23.59 & 8.29 & 30 \\
$\begin{array}{l}\text { Female } \\
\text { Offspring }\end{array}$ & 24.04 & $\left.\right|^{*}$ & 8.53 & 21 \\
Male & 18.18 & 14.36 & 24 \\
$\quad$ Female & 23.97 & 3.83 & 107 \\
& 18.13 & 9.89 & 108 \\
\hline
\end{tabular}

parents were computed to estimate the heritability of bill colour.

\section{Heritability estimates}

The heritability estimates from regressions of offspring on single-parents ranged from $h^{2}=0.34$ to $h^{2}=0.73$ (Fig. 1). The heritability estimates from regressions of sons on fathers, daughters on fathers and sons on mothers were significantly greater than zero but the heritability estimate from the regression of daughters on mothers was not significant (Fig. 1). The arithmetic average for all of these parent-offspring heritability estimates is $h^{2}( \pm 2$ S.E. $)=0.56( \pm 0.49)$.

The ML heritability estimate was significantly greater than zero for male offspring but not female offspring (Table 2). The ML analyses produced results which included negative estimates of dominance genetic variance for both male and female offspring.

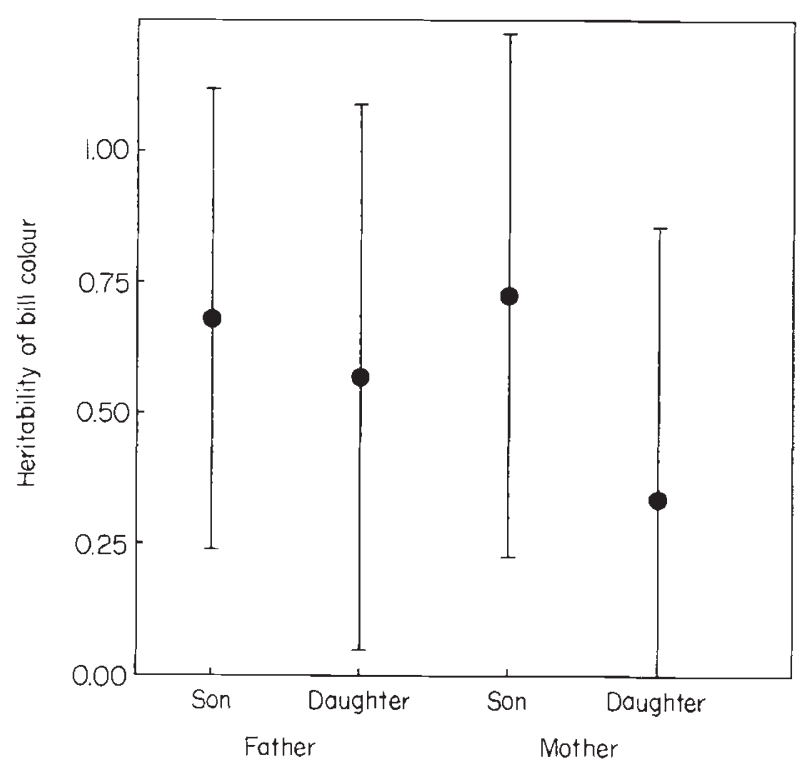

Fig. 1 Heritability estimates $( \pm 2$ S.E.) for zebra finch bill colour. The heritability estimates and standard errors were adjusted for the weak negative assortative mating between the parents. The father-daughter heritability estimate and standard error was multiplied by the bill colour standard deviation ratio between male and female reproductive adults. The mother-son heritability estimate and standard error was multiplied by the inverse of this standard deviation ratio (see Methods). The heritability estimates, significances and numbers of families used in the analyses are as follows: father-son: $h^{2}=0.66, P=0.007, N=20$; father-daughter: $h^{2}=0.54, P=0.045, N=21$; mother-son: $h^{2}=0.70$, $P=0.009, N=22$; mother-daughter: $h^{2}=0.33, P=0.205$, $N=22$. There was a total of 107 male and 108 female offspring in these families. 
Table 2 Quantitative genetic analysis of offspring bill colour using maximum likelihood methods. The additive genetic $\left(V_{\mathrm{A}}\right)$, dominance genetic $\left(V_{\mathrm{D}}\right)$, environmental $\left(V_{\mathrm{E}}\right)$ and phenotypic $\left(V_{\mathrm{P}}\right)$ variance estimates are presented for the maximum likelihood (ML) and restricted maximum likelihood (REML) analyses. The heritability $\left.\left(h^{2}\right)=\left(V_{\mathrm{A}} / V_{\mathrm{P}}\right) / 1+r\right)$ where $r$ is the assortative mating of the parents $(r=-0.23$ for parents of male offspring; $r=-0.15$ for parents of female offspring). The test statistic is twice the difference in the log-likelihoods of the model constraining $V_{\mathrm{A}}=0$ and the model with an unconstrained $V_{\mathrm{A}}$. This statistic is compared to a $\chi^{2}$ distribution with 1 degree of freedom $\left(^{*}-P<0.025\right.$; ns $P>0.05)$

\begin{tabular}{lcccccc}
\hline & $V_{\mathrm{P}}$ & $V_{\mathrm{A}}$ & $V_{\mathrm{D}}$ & $V_{\mathrm{E}}$ & $h^{2}$ & Test of $h^{2}$ \\
\hline ML estimates & & & & & & \\
$\quad$ Female & 9.82 & 4.70 & -1.66 & 6.78 & 0.56 & $1.84^{\text {ns }}$ \\
$\quad$ Male & 4.08 & 6.18 & -9.58 & 7.48 & 1.97 & $7.46^{*}$ \\
$\quad$ REML estimates & & & & & & \\
$\quad$ Females & 9.83 & 4.00 & 0 & 5.83 & 0.48 & $5.52^{*}$ \\
$\quad$ Males & 3.96 & 1.37 & 0 & 2.59 & 0.45 & $2.36^{\text {ns }}$ \\
\hline
\end{tabular}

Therefore, REML analyses were also performed to constrain the dominance genetic variance to zero. The REML heritability estimate was significantly greater than zero for females but not males (Table 2).

\section{Genetic covariance between the sexes}

The uncorrected genetic correlation between male and female bill colour was computed from the covariances between offspring and adult bill colours and found to be $r_{\mathrm{g}}( \pm 2$ S.E. $)=0.82( \pm 0.22)$. These covariances were corrected for the observed negative assortative mating and differences in the variances between male and female bill colour, resulting in $r_{\mathrm{g}}( \pm 2$ S.E. $)=0.91$ $( \pm 0.12)$. The large genetic correlation between male and female bill colour indicates that a large proportion of the genetic variance in bill colour is under common genetic control in males and females.

\section{Discussion}

\section{Potential biases in genetic estimates}

The estimates of bill colour heritabilities in this experiment may underestimate the actual heritability for several reasons. First, extra-pair fertilizations and intraspecific brood parasitism should decrease heritability estimates. Intraspecific brood parasitism should decrease all heritability estimates and extra-pair copulations should decrease only heritability estimates from offspring on father regressions (Alatalo et al., 1984). Based on restriction fragment length polymorphism analyses, the intraspecific brood parasitism rate was higher than the extra-pair copulation rate in this experiment (Burley \& Price, unpublished data). The arithmetic mean of heritability estimates from offspring on father regressions $\left(h^{2}=0.60\right)$ is actually greater than the heritability estimates from offspring on mother regressions $\left(h^{2}=0.52\right)$ which further indicates that extra-pair copulations do not appreciably lower these heritability estimates. Likewise, the genetic correlation between the sexes should not be appreciably affected by extra-pair copulations or intraspecific brood parasitism since extra-pair copulations will reduce both the covariances beween son and father and between daughter and father, and intraspecific brood parasitism will reduce all offspring-parent covariances.

Secondly, any deviation from perfect measurement repeatability could also reduce heritability estimates (Boake, 1989). Measurement repeatability was not estimated in this experiment, but previous short-term repeatability estimates ranged from $r=0.74$ to $r=0.91$ in wild birds (Burley et al., 1992). Bill colour repeatability estimated on long-term data collected both in the field and in the laboratory ranged from $r=0.36$ to $r=0.81$. The lower repeatabilities over long time periods indicate that there was greater environmental variance possibly due to season effects (Burley et al., 1992). In addition, bill colour declined over the course of a breeding season in the wild and during a long-term breeding experiment in the lab (Burley et al., 1992). Such seasonal or breeding influences should have little effect on the founders' bill colours in this experiment because none of the founders had the opportunity to breed before this experiment and all founders were measured on the same day. Offspring bill colour was measured over approximately 19 months and any potential environmental changes in the laboratory over 
this time may have reduced the heritabilities and genetic correlations reported here. These seasonal and/or breeding effects on bill colour repeatability may be important for mate choice when breeding continues for multiple clutches. Adult males that have recently produced fledglings may be expected to have less red bills and, thus, may be less attractive than males that have not recently produced offspring. In addition, environmental factors that occur in nature, but not the laboratory, could decrease estimates of bill colour heritability in natural populations compared with laboratory populations.

On the other hand, common-environmental effects might inflate these heritability values. If, for example, the parents with redder bills were in superior condition and provided better care for their offspring, and if offspring that experienced better care developed redder bills, the environmental correlation between offspring and parent bill colour would be positive, leading to inflated heritability estimates. The similarity of heritability estimates from offspring on parent regressions and REML analyses of full- and half-siblings (Fig. 1, Table 2) suggests that a common-environmental factor was not appreciably affecting the bill colour heritability estimates in this study. In addition, the negative dominance genetic variance from the ML analyses (Table 2) indicates that full-siblings were not phenotypically more similar than expected relative to half-siblings. This negative dominance genetic variance estimate may also contain environmental factors that reduce the phenotypic similarity of full-siblings. Results from a cross-fostering experiment also yielded significant heritability estimates of bill colour and indicate that common-environmental effects which could have increased the similarity of parents and offspring were minor (Price, 1991).

\section{Genetic estimates}

Additive genetic variance explained approximately 58 per cent of the phenotypic variance in bill colour (Fig. 1 , Table 2). The maximum likelihood analyses indicated that the dominance genetic variance was low (Table 2). The documentation of moderately large heritability estimates for sexually selected traits in the zebra finch and several other species (Cade, 1981; Carson \& Teramoto, 1984; Butlin \& Hewitt, 1986; Simmons, 1987; Hedrick, 1988; McLain, 1987; Sakaluk \& Smith, 1988; Moore, 1989; Houde, 1992) indicates that the potentially strong selection pressure exerted by females choosing mates has not eliminated the genetic variation of attractive traits. Therefore, attractive traits may be evolving in natural populations.
The genetic correlation in this experiment indicates that approximately 90 per cent of the additive genetic variance in bill colour is controlled by the same genes in males and females. The occurrence of a substantial positive genetic correlation between the sexes can constrain bill colour evolution slowing the rate at which bill colour evolves to sex-specific optima (Lande, 1980a, 1987; Lande \& Arnold, 1985). In this same experiment, there was significant opposing selection on male and female bill colour that is consistent with zebra finch bill colour being displaced from sex-specific optima (Price, 1991). In other species, estimates of genetic correlations between the sexes have also been very high for morphological characters (Harrison, 1953; Frankham, 1968a, 1968b; Crowley \& Atchley, 1986; Crowley et al., 1986; Rogers \& Mukherjee, 1992). Therefore, genetic covariances between traits expressed in males and females may cause traits to be displaced from their sex-specific optima in other species for evolutionarily long time periods (Lande, 1980a).

\section{Evolution of sexual dichromatism and the constancy of genetic covariances and selection intensities}

Traditionally, it has been thought that the opportunity for sexual selection and thus the potential for sexual dichromatism is greater in polygynous species because the ratio of the variances of male mating success to female mating success is greater than in monogamous species (Selander, 1972; Payne, 1984). However, comparative analyses indicate that the degree of plumage dichromatism is not correlated with the mating system of warblers (Shutler \& Weatherhead, 1990) or with the occurrence of lek mating systems in nine avian families (Hoglund, 1989). These surprising results have been interpreted either as being due to other selective pressures on male plumage colour that are unrelated to the mating system (Shutler \& Weatherhead, 1990) or as evidence that plumage colour is currently not sexually selected (Hoglund, 1989). Our results, however, suggest that genetic covariances between the sexes may limit the evolutionary response of sexual dichromatism in taxa that experience different intensities of sexual selection in each sex. The comparative analyses described above are based upon the implicit assumption that the genetic variance within the sexes and covariances between the sexes for colour traits remain historically constant across the taxa being studied. Polygynous and monogamous species may experience different degrees of sexual selection yet exhibit similar degrees of sexual dichromatism because of differences in the genetic variance and covariance among species (Cheverud et al., 1985). Very little is known about the 
inheritance of colour traits but it is possible that different colour traits (e.g. different hues and the size and location of colour patches) will exhibit varying degrees of genetic (co)variances within and between taxa. Therefore, a detailed knowledge of the genetic variance and covariance of colour traits is necessary before the importance of mating systems to influence the evolution of colour traits can be evaluated.

Lande (1980a, 1987) and Lande \& Arnold (1985) predicted that the evolution of sexually dimorphic traits may be characterized by two evolutionary phases. In the first phase, the trait in both sexes evolves in the same direction until a joint optimum is reached in which the selection in one sex is similar in magnitude to but opposite in sign from selection in the other sex. In the second phase, the traits in the sexes evolve in opposite directions until the sex-specific optima are reached. This second phase is characterized by a high genetic covariance between male and female traits and opposing selection on the sexes and may be several orders of magnitude longer than the first phase (Lande, 1980a, 1987). Because this study of zebra finch bill colour has demonstrated a high genetic covariance and we have evidence of opposing selection between the sexes (Price, 1991), we believe that zebra finch bill dichromatism may be in the second phase of evolution towards dimorphism. If large genetic covariances between the sexes commonly occur for colour traits in other species, then many species can be expected to be displaced from their sex-specific optima and to experience opposing selection between males and females for colour traits.

\section{Acknowledgements}

We thank C. R. B. Boake, B. Flesher, I. L. Heisler and K. Spitze for discussion of this research and critical comments on earlier drafts of this paper. E. Bridgett, L. Petersen and EEE 290 students provided much-appreciated assistance. Research was supported by NSF grants BSR 88-17977 and BSR 89-01345 and University of Illinois Graduate College Grant and Summer Fellowship. D.K.P. was supported by a NIH Ethology Postdoctoral Training Grant at the University of Tennessee during the preparation of this manuscript. The research reported in this paper constitutes partial fulfilment of the Ph.D. in Biology at the University of Illinois for D.K.P.

\section{References}

ALATAlo, R. V., GUSTAFsSON, L. AND LUNDBERG, A. 1984. High frequency of cuckoldry in pied and collared flycatchers. $\mathrm{Oi}$ kos, 42, 41-47.
BECKER, W. A. 1985. Manual of Quantitative Genetics, 4th edn. Academic Enterprises Press, Pullman, Washington.

BOAKE, C. R. B. 1989. Repeatability: its role in evolutionary studies of mating behavior. Evol. Ecol., 3, 173-182.

BURLEY, N. 1985. The organization of behavior and the evolution of sexually selected traits. In: Gowaty, P. A. and Mock, D. W. (eds) Avian Monogamy, American Ornithologists' Union, Washington, D.C.

BURLEY, N. AND COOPERSMITH, C. B. 1987. Bill color preferences of zebra finches. Ethology, 76, 133-151.

BURLEY, N., PRICE, D. K. AND ZANN, R. 1992. Bill color, reproduction and condition effect in wild and domesticated zebra finches. Auk, 109, 13-23.

BUTCHER, G. S. AND ROHWER, S. 1989. The evolution of conspicuous color and distinctive coloration for communication in birds. In: Power, D. M. (ed.) Current Ornithology, vol. 6, Plenum Press, New York, N.Y.

BUTLIN, R. K. AND HEWITT, G. M. 1986. Heritability estimates for characters under sexual selection in the grasshopper, Chorthippus brunneus. Anim. Behav., 34, 1256-1261.

CADE, w. 1981. Alternative male strategies: Genetic differences in crickets. Science, 212, 563-564.

CARSON, H. L. AND TERAMOTO, L. T. 1984. Artificial selection for a secondary sexual character in males of Drosophila silvestris from Hawaii. Proc. Natl. Acad. Sci. U.S.A., 81, 3915-3917.

CHARLESWORTH, B. 1988. The evolution of mate choice in a fluctuating environment. J. Theoret. Biol., 130, 191-204.

CHEVERUD, J. M., DOW, M. M. AND LEUTENEGGER, w. 1985. The quantitative assessment of phylogenetic constraints in comparative analyses: sexual dimorphism in body weight among primates. Evolution, 39, 1335-1351.

CROWLEY, D. E. AND ATCHLEY, W. R. 1988. Quantitative genetics of Drosophila melanogaster. II Heritabilities and genetic correlations between sexes for head and thorax traits. Genetics, 119, 421-433.

CROWLEY, D. E., ATCHLEY, W. R. AND RUTLEDGE, J. J. 1986. Quantitative genetics of Drosophila melanogaster. I. Sexual dimorphism in genetic parameters for wing traits. Genetics, 114, 549-566.

DARWIN, C. 1871. The Descent of Man and Selection in Relation to Sex. John Murray, London, U.K.

EISEN, E. J. AND LEGATES, J. E. 1966. Genotype-sex interaction and the genetic correlation between the sexes for body weight in Mus musculus. Genetics, 54, 611-623.

EISEN, E. J. AND haNRAhAN, J. P. 1972. Selection for sexual dimorphism in body weight of mice. Aust. J. Biol. Sci., 25, 1015-1024.

FALCONER, D. S. 1981. Introduction to Quantitative Genetics, 2nd edn. Longman Press, London, U.K.

FISHER, R. 1958. The Genetical Theory of Natural Selection, 2nd edn. Clarendon Press, Oxford, U.K.

FRANKHAM, R. 1968a. Sex and selection for a quantitative character in Drosophila I. Single-sex selection. Aust. J. Biol. Sci., 21, 1215-1223.

FRANKHAM, R. 1968b. Sex and selection for a quantitative character in Drosophila II. The sex dimorphism. Aust. J. Biol. Sci., 21, 1225-1237.

Goodwin, D. 1982. Estrildid Finches of the World, Cornell University Press, Ithaca, N.Y. 
HARRISON, B. J. 1953. Reversal of a secondary sex character by selection. Heredity, 7, 153-164.

HEDRICK, A. v. 1988. Female choice and the heritability of attractive male traits: an empirical study. Amer. Natur., 132, 267-276.

HOUDE, A. E. 1992. Sex-linked heritability of a sexually selected character in a natural population of Poecilia reticulata (Pisces: Poeciliidae) (guppies). Heredity, 69, 229-235.

HILL, W. G. AND THOMPSON, R. 1977. Design of experiments to estimate offspring-parent regression using selected parents. Anim. Prod., 24, 163-168.

HoGLUND, J. 1989. Size and plumage dimorphism in lekbreeding birds: a comparative analysis. Amer. Natur., 134, 72-87.

1mmelmann, K. 1965. Australian Finches in Bush and Aviary. Halstead Press, Sydney, Australia.

KIRKPATR1CK, M. 1987. Sexual selection by female choice in polygynous animals. An. Rev. Ecol. Syst., 18, 43-70.

KIRKPATR1CK, M. AND RYAN, M. J. 1991. The evolution of mating preferences and the paradox of the lek. Nature, 350, 33-38.

LANDE, R. 1980a. Sexual dimorphism, sexual selection, and adaptation in polygenic characters. Evolution, 34, 292-305.

LANDE, R. 1980b. The genetic covariance between characters maintained by pleiotropic mutations. Genetics, 94, 203-215.

LANDE, R. 1981. Models of speciation by sexual selection on polygenic traits. Proc. Natl. Acad. Sci. U.S.A., 78, 3721-3725.

LANDE, R. 1987. Genetic correlations between the sexes in the evolution of sexual dimorphism and mating preferences. In: Bradbury, J. W. and Andersson, M. (eds) Sexual Selection: Testing the Alternatives, Dahlem Konferensen, Wiley Press, Chichester, U.K.

LANDE, R. AND ARNOLD, S. J. 1985. Evolution of mating preference and sexual dimorphism. J. Theoret. Biol., 117, 651-664.

LYNCH, M. 1988. The rate of polygenic mutation. Genet. Res., 51, 137-148.
McLAIN, D. K. 1987. Heritability of size, a sexually selected character, and the response to sexual selection in a natural population of the southern green stink bug, Nezara viridula (Hemiptera: Pentatomidae). Heredity, 59, 391-395.

MOORE, A. J. 1989. The inheritance of social dominance, mating behavior, and attractiveness to mate in male Nauphoeta cinerea. Anim. Behav., 39, 388-397.

PAYNE, R. B. 1984. Sexual Selection, Lek and Arena Behavior, and Sexual Dimorphism in Birds. Ornithological Monographs, 33, 1-52.

PRICE, D. K. 1991. Constraints on the evolution of attractive traits: natural selection, sexual selection and quantitative genetics in the zebra finch. Ph.D Thesis, University of Illinois at Urbana-Champaign.

POM1ANKOWSK1, A. 1988. The evolution of female mate preferences for male quality. In: Harvey, P. H. and Partridge, L. (eds) Oxford Surveys in Evolutionary Biology, vol. 5, Oxford University Press, Oxford, U.K.

REEVE, C. R. 1961. A note on non-random mating in progeny tests. Genet. Res. Camb., 2, 195-203.

ROGERS, A. R. AND MUKHERJEE, A. 1992. Quantitative genetics of sexual dimorphism in human body size. Evolution, 46, 226-234.

SAKALUK, S. K. AND SMITH, R. L. 1988. Inheritance of male parental investment in an insect. Amer. Natur., 132, 594-601.

SELANDER, R. K. 1972. Sexual selection and dimorphism in birds. In: Campbell, B. (ed.) Sexual Selection and the Descent of Man, 1871-1971, Aldine Press, Chicago, Il.

SHAw, R. 1987. Maximum-likelihood approaches applied to quantiative genetics of natural populations. Evolution, 41, 812-826.

SHUTLER, D. AND WEATHERHEAD, P. J. 1990. Targets of sexual selection: song and plumage of wood warblers. Evolution, 44, 1967-1977.

SIMMONS, L. w. 1987. Heritability of a male character chosen by females of the field cricket (Gryllus bimaculatis). Behav. Ecol. Sociobiol., 21, 129-133.

SLATKIN, M. 1984. Ecological causes of sexual dimorphism. Evolution, 38, 622-630. 